\title{
Non-anti-commutative Deformation of Complex Geometry
}

\author{
Sergei V. Ketov \\ Department of Physics, Tokyo Metropolitan University, Hachioji-shi, Tokyo \\ 192-0397, Japan; ketov@phys.metro-u.ac.jp
}

Summary. In this talk I review the well known relation existing between extended supersymmetry and complex geometry in the non-linear sigma-models, and then briefly discuss some recent developments related to the introduction of the nonanti-commutativity in the context of the supersymmetric non-linear sigma-models formulated in extended superspace. This contribution is suitable for both physicists and mathematicians interesting in the interplay between geometry, supersymmetry and non(anti)commutativity.

\subsection{Introduction}

Being a theoretical physicist, one gets used to mathematical tools, whose role in modern theoretical high-energy physics is indispensable and indisputable. Especially when the experimental base is limited or does not exist, the use of advanced mathematics to get new insights in physics is particularly popular. So it is no surprise that mathematical knowledge of theoretical physicists is quite high. However, the way of dealing with mathematics in theoretical physics is different from that commonly used by mathematicians, and even the motivation and goals are different, as is also well known. I would like to draw attention to another, less known fact that physical considerations may sometimes lead to new mathematics, or rediscovery of some famous mathematical facts. In my contribution to this workshop, aimed towards more cooperation and understanding between physicists and mathematicians, I would like to explain how investigation of supersymmetry in field theory of the non-linear sigma-models might have led to rediscovery of complex geometry and related mathematical tools. In addition, I would like to explain how introducing more fundamental structure (namely, non-anti-commutativity in superspace) leads to a new deformation of complex geometry, whose geometrical significance is yet to be understood.

The paper is organized as follows. In sect. 2 the basic notions of the nonlinear sigma-models are introduced. My presentation is 'minimal' on purpose, 
without going into details and/or many generalizations that might be easily added. I just summarize the basic ideas. In sect. 3, I introduce a simple superspace, and review the known relation between extended supersymmetry and complex geometry in the non-linear sigma-models, by getting all basic notions of complex geometry from a single and straightforward fieldtheoretical calculation. In sect. 4 some extended superspace techniques, making extended supersymmetry to be manifest, are briefly discussed. In sect. 5 some more superspace structure is added by introducing the notion of NonAnti-Commutativity (NAC) or 'quantum superspace', and its impact on the non-linear sigma-model target space is calculated. The simplest non-trivial explicit example of the NAC-deformed $C P(1)$ metric is given in sect. 6. Our conclusion is sect. 7 .

\subsection{Non-linear sigma-models}

The Non-Linear Sigma-Model (NLSM) is a scalar field theory whose (multicomponent) scalar field $\phi^{a}\left(x^{\mu}\right)$ is defined in a $d$-dimensional 'spacetime' or a 'worldvolume' parametrized by local coordinates $\left\{x^{\mu}\right\}, \mu=1,2, \ldots, d$. The fields $\phi^{a}$ take their values in a $D$-dimensional Riemannian manifold $M$, called the NLSM target space, $a=1,2, \ldots, D$. The NLSM field values $\phi^{a}$ can thus be considered as a set of (local) coordinates in $M$, whose metric is fielddependent. The NLSM format is the very general field-theoretical concept whose geometrical nature is the main reason for many useful applications of NLSM in field theory, string theory, condensed matter physics and mathematics (see e.g., the book [1] for much more).

We assume the NLSM spacetime or worldvolume to be flat Euclidean space $R^{d}$, for simplicity, so that the NLSM action is supposed to be invariant under translations (with generators $P_{\mu}$ ) and rotations (with generators $M_{\mu \nu}$ ) in $R^{d}$. Let $d s^{2}=g_{a b}(\phi) d \phi^{a} d \phi^{b}$ be a metric in $M$. Then a generic NLSM action is given by

$$
S_{\text {bos. }}[\phi]=\int d^{d} x L\left(\partial_{\mu} \phi, \phi\right), \quad L=\frac{1}{2} g_{a b}(\phi) \delta^{\mu \nu} \partial_{\mu} \phi^{a} \partial_{\nu} \phi^{b}+m^{2} V(\phi),
$$

where summation over repeated indices is always implied. The function $V(\phi)$ is called a scalar potential in field theory with a mass parameter $m$. The higher derivatives of the field $\phi$ are not allowed in the Lagrangian $L$, with the notable exception of $d=2$ where an extra (Wess-Zumino) term may be added to eq. (1.1):

$$
L_{\mathrm{WZ}}=\frac{1}{2} b_{a b}(\phi) \varepsilon^{\mu \nu} \partial_{\mu} \phi^{a} \partial_{\nu} \phi^{b} .
$$

The 2-form $B=b_{a b}(\phi) d \phi^{a} \wedge d \phi^{b}$ in eq. (1.2), is called a torsion potential in $M$, by the reason to be explained in the next sect. 1.3. In string theory, it is called a $B$-field (or a Kalb-Ramond field). 


\subsection{Supersymmetric NLSM}

There are two different ways to supersymmetrize the NLSM: either in the worldvolume, or in the target space. Here we only discuss the worldvolume supersymmetrization of NLSM, in the case of even $d .{ }^{1}$ Then adding supersymmetry amounts to the extension of the Euclidean space motion group $S O(d) \times T^{d}$ to a supergroup, with the key superalgebra relation

$$
\left\{Q_{\alpha}^{i}, \bar{Q} \dot{\beta}_{j}\right\}_{+}=\underset{\alpha \sigma_{\dot{\beta}}}{\mu} P_{\mu} \delta^{i}{ }_{j},
$$

where the additional fermionic supercharges $Q$ and $\bar{Q}$ are chiral and anti-chiral spinors of $S O(d)$, repectively, in the fundamental representation of the internal $U(N)$ symmetry group, denoted by latin indices $i, j=1,2, \ldots, N$. The $N$ here is a number of supersymmetries, so that the $N>1$ supersymmetry is called the extended one. The chiral $\sigma$-matrices in eq. (1.3) obey Clifford algebra in $d$ dimensions.

As regards the NLSM, it is not difficult to demonstrate by using only group-theoretical arguments that $d \leq 6$ [1], and when $\mathrm{d}=2$ then $N \leq 4$ [2].

The model-independent technology for a construction of off-shell manifestly supersymmetric field theories is called superspace. To give an example, let us consider the simplest case of the $N=1$ supersymmetric NLSM in $d=2$. The two-dimensional complex coordinates $z$ and $\bar{z}$ can be extended by the anti-commuting (Grassmann) fermionic (spinor) coordinates $\theta$ and $\bar{\theta}$ to form a superspace $(z, \bar{z}, \theta, \bar{\theta})$. Tensor functions in superspace are called superfields. A superfield is always equivalent to a supermultiplet of the usual fields, e.g.

$$
\Phi(z, \bar{z}, \theta, \bar{\theta})=\phi+\theta \psi+\bar{\theta} \bar{\psi}+\bar{\theta} \theta F
$$

in terms of the bosonic field components $\phi(z, \bar{z})$ and $F(z, \bar{z})$, and the fermionic field components $\psi(z, \bar{z})$ and $\bar{\psi}(z, \bar{z})$.

The supercharges can be easily realized in superspace as the differential operators

$$
Q=\frac{\partial}{\partial \theta}-\theta \partial, \quad \text { and } \quad \bar{Q}=\frac{\partial}{\partial \bar{\theta}}-\bar{\theta} \bar{\partial},
$$

where we have introduced the notation $\partial=\partial_{z}$ and $\bar{\partial}=\partial_{\bar{z}}$. It is not difficult to check that the covariant derivatives

$$
D=\frac{\partial}{\partial \theta}+\theta \partial, \quad \bar{D}=\frac{\partial}{\partial \bar{\theta}}+\bar{\theta} \bar{\partial}, \quad \partial \quad \text { and } \quad \bar{\partial},
$$

all (anti)commute with the supercharges (1.5) indeed, which allows us to use them freely in the covariant superspace action. Then the unique $\mathrm{N}=1$ supersymmetric extension of the bosonic NLSM action (1.1) is easily constructed in $\mathrm{N}=1$ superspace as follows (we ignore a scalar potential here):

\footnotetext{
${ }^{1}$ In string theory, the world-sheet supersymmetrization is known as the NeveuSchwarz-Ramond (NSR) approach, whereas the target space supersymmetrization is called the Green-Schwarz (GS) approach.
} 


$$
S_{1}=\int d^{2} x d^{2} \theta\left(g_{a b}+b_{a b}\right) D \Phi^{a} \bar{D} \Phi^{b},
$$

where both $g_{a b}(\Phi)$ and $b_{a b}(\Phi)$ are now functions of the superfields $\Phi^{a}$ of eq. (1.4). The component fields $F$ appear to be non-propagating (they are called to be auxiliary), since they satisfy merely algebraic equations of motions. They are supposed to be substituted by solutions to their 'equations of motion'. Having evaluated the Berezin integral in eq. (1.7), one gets eq. (1.1) as the only purely bosonic contribution that is modified by the fermionic terms, namely, by a sum of the covariant Dirac term and the quartic fermionic interaction whose field-dependent couplings are given by the curvature tensor with torsion.

The $B$-field, in fact, enters the field action $S_{1}$ only via its curl (= torsion in $M$ )

$$
T^{a}{ }_{b c}=-\frac{3}{2} g^{a d} b_{[b c, d]},
$$

that, in its turn, enters the action only via the connections (in $M$ )

$$
\Gamma_{ \pm b c}^{a}=\left\{\begin{array}{c}
a \\
b c
\end{array}\right\} \pm T_{b c}^{a} .
$$

By construction the two-dimensional action $S_{1}$ is invariant under the $\mathrm{N}=1$ supersymmetry transformations

$$
\delta_{\text {susy }} \Phi^{a}=\varepsilon Q \Phi^{a}+\bar{\varepsilon} \bar{Q} \Phi^{a},
$$

with

$$
Q \Phi^{a} \mid=\psi^{a} \quad \text { and } \quad \bar{Q} \Phi^{a} \mid=\bar{\psi}^{a},
$$

where $\mid$ denotes the leading (i.e. $\theta$ - and $\bar{\theta}$ - independent) part of a superfield, while $\varepsilon$ and $\bar{\varepsilon}$ are the infinitesimal fermionic (Grassmann) N=1 supersymmetry transformation parameters. Eq. (1.11) can serve as the definition of the fermionic superpartners of the bosonic NLSM field $\phi^{a}$.

It is not difficult to generalize the NLSM (1.7) by adding a scalar superpotential in superspace,

$$
S_{\text {pot. }}=m \int d^{2} x d^{2} \theta W(\Phi),
$$

with a arbitrary real function $W(\Phi)$ and a mass parameter $m$. It gives rise to the scalar potential

$$
V(\phi)=\frac{1}{2} m^{2} g^{a b}(\phi) \partial_{a} W(\phi) \partial_{b} W(\phi),
$$

while it does not modify the NLSM kinetic terms, as is already clear from dimensional reasons.

A generic two-dimensional NLSM with an arbitrary Riemannian target space $M$ (and no scalar potential) can always be $\mathrm{N}=1$ supersymmetrized, 
as in eq. (1.7). When $M$ is a Lie group manifold, there is a preferred (groupinvariant) choice for its metric and torsion, while such NLSM is called a WessZumino-Novikov-Witten (WZNW) model [1]. One may also introduce the socalled gauged WZNW models with a homogeneous target space $G / H$, where $H$ is a subgroup of $G$. In differential geometry, it corresponds to the quotient construction [1].

The next relevant question is: which restrictions on the NLSM target space $M$, in fact, imply more supersymmetry, i.e. $N>1$ ? To answer that question, all one needs is to write down the most general Ansatz for the second supersymmetry transformation law (it follows by dimensional reasons) in terms of the $\mathrm{N}=1$ superfields as

$$
\delta_{2} \Phi=\eta J_{b}^{a}(\Phi) D \Phi^{b}+\bar{\eta} \bar{J}_{b}^{a}(\Phi) \bar{D} \Phi^{b},
$$

and then impose the invariance condition

$$
\delta_{2} S_{1}=0 .
$$

In equation (1.14), the $\eta$ and $\bar{\eta}$ are the infinitesimal parameters of the second supersymmetry, while $J^{a}{ }_{b}(\Phi)$ and $\bar{J}_{b}{ }_{b}(\Phi)$ are some tensor functions to be fixed by eq. (1.15). It is straightforward (though tedious) to check that the condition (1.14) amounts to the following restrictions (see e.g., ref. [3]):

$$
\nabla_{c}^{+} J_{b}^{a}=\nabla_{c}^{-} \bar{J}_{b}^{a}=0,
$$

and

$$
g_{b c} J_{a}^{c}=-g_{a c} J_{b}^{c}, \quad g_{b c} \bar{J}_{a}^{c}=-g_{a c}{\overline{J^{c}}}_{b} .
$$

In addition, one gets the standard (on-shell) $\mathrm{N}=2$ supersymmetry algebra (1.3) provided that (see e.g., ref. [3])

$$
J^{2}=\bar{J}^{2}=-\mathbf{1} \quad \text { and } \quad N_{b c}^{a}[J, J]=N_{b c}^{a}[\bar{J}, \bar{J}]=0,
$$

where we have introduced the Nijenhuis tensor

$$
N_{b c}^{a}[A, B]=A_{[b}^{d} B_{c], d}^{a}+A_{d}^{a} B^{d}{ }_{[b, c]}+B^{d}{ }_{[b} A_{c], d}^{a}+B^{a}{ }_{d} A_{[b, c]}^{d} .
$$

So we can already recognize (or re-discover) the basic notions of (almost) complex geometry, such as an (almost) complex structure, a hermitean metric, a covariantly constant (almost) complex structure, and an integrable complex structure (see e.g., ref. [4]). To be precise, we get the following theorem:

a two-dimensional $N=1$ supersymmetric $N L S M$ is actually (on-shell) $N=2$ supersymmetric, if and only if (1) it allows two (almost) complex structures, $J$ and $\bar{J}$, (2) the NLSM metric is hermitean with respect to each of them, and (3) each (almost) complex structure is covarianlty constant with respect to the asociated $( \pm)$ connection, so that it is actually integrable.

The integrability here means the existence of holomoprhic and antiholomorphic coordinates (i.e. the holomorphic transitions functions) after 
rewriting a complex structure to the diagonal form (with the eigenvalues $i$ and $-i)$.

It should be noticed that the complex structures $J$ and $\bar{J}$ may not be commuting with each other, $[J, \bar{J}] \neq 0$, because they are covariantly constant with respect to the different connections in eq. (1.9), respectively. For instance, the mixed $\mathrm{N}=2$ supersymmetry commutator

$$
[\delta(\eta), \delta(\bar{\eta})] \Phi^{a}=\eta \bar{\eta}[J, \bar{J}]_{b}^{a}\left(D \bar{D} \Phi^{b}+\Gamma_{-c d}^{b} D \Phi^{d} \bar{D} \Phi^{c}\right),
$$

is required to be vanishing by the $\mathrm{N}=2$ supersymmetry algebra (1.3). It is already true on-shell, i.e. when the NLSM equations of-motions, $D \bar{D} \Phi^{b}+$ $\Gamma^{b}{ }_{-c d} D \Phi^{d} \bar{D} \Phi^{c}=0$, are satisfied, though it is also the case off-shell only if $[J, \bar{J}]=0$. The complex structures $J$ and $\bar{J}$ may not therefore be simultaneously integrable, in general. If, however they do commute, then the existence of an off-shell $\mathrm{N}=2$ extended superspace formulation of such $\mathrm{N}=2 \mathrm{NLSM}$ with manifest $\mathrm{N}=2$ supersymmetry is guaranteed.

\subsection{NLSM in extended superspace}

To give the simplest example of the $\mathrm{N}=2$ extended superspace in two dimensions $(z, \bar{z})$, let's introduce two (Grassmann) fermionic coordinates for each chirality, i.e. $\left(z, \theta^{+}, \theta^{-}\right)$and $\left(\bar{z}, \bar{\theta}^{+}, \bar{\theta}^{-}\right)$, and then the $\mathrm{N}=2$ supercharges $Q_{ \pm}$ and $\bar{Q}_{ \pm}$, the $\mathrm{N}=2$ superspace covariant derivatives $D_{ \pm}$and $\bar{D}_{ \pm}$, and $\mathrm{N}=2$ scalar superfields $\Phi^{i}\left(z, \bar{z}, \theta^{+}, \theta^{-}, \bar{\theta}^{+}, \bar{\theta}^{-}\right)$, like in the $\mathrm{N}=1$ case (see the previous section), where now $i=1,2, \ldots, m$.

However, there is the immediate problem: a general (unconstrained) $\mathrm{N}=2$ scalar superfield has a physical vector field component that is not suitable for the NLSM. The simplest way to remedy that problem is to use the (off-shell) $\mathrm{N}=2$ chiral and anti-chiral superfields, subject to the constraints

$$
\bar{D}_{ \pm} \Phi=0 \quad \text { and } \quad D_{ \pm} \bar{\Phi}=0
$$

respectively. Their most general NLSM action is then given by

$$
S_{2}=\int d^{2} x d^{2} \theta d^{2} \bar{\theta} K(\Phi, \bar{\Phi})+m \int d^{2} x d^{2} \theta W(\Phi)+m \int d^{2} x d^{2} \bar{\theta} \bar{W}(\bar{\Phi}),
$$

in terms of a non-holomorphic kinetic potential $K(\Phi, \bar{\Phi})$ and a holomorphic superpotential $W(\Phi)$.

A simple straightforward calculation of the NLSM metric out of eq. (1.22) reveals a Kähler metric $g_{i \bar{j}}=\partial_{i} \bar{\partial}_{j} K$ with the Kähler potential $K$, and no torsion. Therefore, Kähler complex geometry could have been also re-discovered from the $\mathrm{N}=2$ supersymmetric NLSM.

When one adds the so-called twisted chiral $\mathrm{N}=2$ superfields, subject to the following off-shell $\mathrm{N}=2$ superspace constraints: 


$$
\bar{D}_{+} \tilde{\Phi}=D_{-} \tilde{\Phi}=0 \quad \text { and } \quad \bar{D}_{-} \overline{\tilde{\Phi}}=D_{+} \overline{\tilde{\Phi}}=0,
$$

their most general $\mathrm{N}=2$ superspace action,

$$
S_{2, T}=\int d^{2} x d^{2} \theta d^{2} \bar{\theta} K(\Phi, \bar{\Phi}, \tilde{\Phi}, \overline{\tilde{\Phi}})+\text { obvious superpotential terms }
$$

would give rise to a non-trivial torsion too, though with the commuting complex structures, $[J, \bar{J}]=0[5]$. Actually, the exchange $\Phi \leftrightarrow \tilde{\Phi}$ corresponds to the T-duality in string theory.

To get the most general $\mathrm{N}=2$ supersymmetric NLSM with $[J, \bar{J}] \neq 0$, one has to add the so-called semi-chiral (reducible) $\mathrm{N}=2$ superfields, $\hat{\Phi}$ and $\overline{\hat{\Phi}}$, subject to the off-shell $\mathrm{N}=2$ superspace constraints [6, 3]

$$
\bar{D}_{+} \bar{D}_{-} \hat{\Phi}=0 \quad \text { and } \quad D_{+} D_{-} \overline{\hat{\Phi}}=0 .
$$

When asking for even more supersymmetry in a two-dimensional supersymmetric NLSM, one gets three linearly independent (almost) complex structures of each chirality, obeying a quaternionic algebra (see e.g., ref. [1]),

$$
J_{a}^{ \pm(A) b} J_{b}^{ \pm(B) c}=-\delta^{A B} \delta_{a}^{c}+\varepsilon^{A B C} J_{a}^{ \pm(C) c}, \quad \text { where } A, B, C=1,2,3,
$$

and similarly for $\bar{J}$. They all must be covariantly constant,

$$
\nabla^{ \pm} J^{ \pm}=0
$$

respectively. In particular, $\mathrm{N}=3$ supersymmetry implies $\mathrm{N}=4$ supersymmetry.

Unfortunately, a geometrical description of the two-dimensional $\mathrm{N}=4$ supersymmetric NLSM with torsion is still incomplete. In the case of the vanishing torsion, an $\mathrm{N}=1$ supersymmetric NLSM is, in fact, $\mathrm{N}=4$ supersymmetric if and only if its target space is hyper-Kähler (see e.g., ref. [1] for more details).

When a supersymmetric NLSM in question is, in fact, a (gauged) WZNW model, then its $\mathrm{N}=4$ supersymmetry implies that its target space must be a product of Wolf spaces [7,3]. The Wolf space can be associated with any simple Lie group $G$. Let $\psi$ be the highest weight root of $G$, and let $\left(E_{\psi \pm}, H\right)$ be the generators of the $s u(2)_{\psi}$ subalgebra of Lie algebra of $G$ (say, in Chevalley basis), associated with $\psi$. Then the Wolf space is given by the coset

$$
\text { Wolf space }=\frac{G}{H_{\perp} \otimes S U(2)_{\psi}},
$$

where we have introduced the $S U(2)_{\psi}$ Lie group of the Lie algebra $s u(2)_{\psi}$ and the centralizer $H_{\perp}$ of the $S U(2)_{\psi}$ in $G$.

An efficient off-shell $\mathrm{N}=4$ superspace description of all two-dimensional $\mathrm{N}=4$ supersymmetric NLSMs does not exist, though the use of harmonic superspace [8] with the infinite number of auxiliary fields may be useful for describing a large class of the manifstly $\mathrm{N}=4$ supersymmetric NLSM. 
It is also worth mentioning that the chiral generators of supersymmetry in two dimensions are independent, so that it is possible to have an unequal number of 'left' and 'right' supersymmetries. It is called 'heterotic' or $(p, q)$ supersymmetry. ${ }^{2}$ It is always possible to construct the minimal or $(1 / 2,0)$ supersymmetric extention of any NLSM. A generic $(1 / 2,1)$ supersymmetric NLSM can be formulated in $(1 / 2,1)$ superspace. Less is known about other $(p, q)$ supersymmetric NLSM with $n=3,4$.

Finally, there is a simple relation between extended supersymmetry and higher $(d>2)$ dimensions, which is just based on the representation theory of spinors and Clifford algebras in various dimensions. A supersymmetric NLSM can first be formulated in six or four dimensions, and then it can be rewritten to lower dimensions, by simply restricting all its fields to be dependent upon lower number of their worldvolume coordinates (this procedure is called dimensional reduction). The manifestly supersymmetric formulation of a higher-dimensional supersymmetric NLSM often requires the use of sophisticated (constrained) superfields [1]. In quantum theory, only two-dimensional NLSM are renormalizable, while their higher-dimensional counterparts are not. The same is true for the supersymmetric NLSM [1].

\subsection{Non-anticommutative deformation of four-dimensional supersymmetric NLSM}

Non-Anti-Commutativity (NAC) or quantum superspace [9] is a natural extension of the ordinary superspace, when the fermionic superspace coordinates are assumed to obey a Clifford algebra instead of being Grassmann (i.e. anticommutative) variables [10]. The non-anticommutativity naturally arises in the D3-brane superworldvolume, in the type-IIB constant Ramond-Ramond type background, in superstring theory [11]. In four dimensions, the NAC deformation is given by

$$
\left\{\theta^{\alpha}, \theta^{\beta}\right\}_{*}=C^{\alpha \beta},
$$

where $C^{\alpha \beta}$ can be identified with a constant self-dual gravi-photon background [11]. The remaining $\mathrm{N}=1$ superspace coordinates in the chiral basis $\left(y^{\mu}=\right.$ $x^{\mu}+i \theta \sigma^{\mu} \bar{\theta}, \mu, \nu=1,2,3,4$ and $\left.\alpha, \beta, \ldots=1,2\right)$ can still (anti)commute,

$$
\left[y^{\mu}, y^{\nu}\right]=\left\{\bar{\theta}^{\dot{\alpha}}, \bar{\theta}^{\dot{\beta}}\right\}=\left\{\theta^{\alpha}, \bar{\theta}^{\dot{\beta}}\right\}=\left[y^{\mu}, \theta^{\alpha}\right]=\left[y^{\mu}, \bar{\theta}^{\dot{\alpha}}\right]=0 .
$$

provided we begin with a four-dimensional Euclidean ${ }^{3}$ worldvolume having the coordinates $x^{\mu}$. A supersymmetric field theory in the NAC superspace was extensively studied in the recent past, soon after the pioneering paper [13]. The choice (1.30) preserves locality in a NAC-deformed field theory.

\footnotetext{
${ }^{2}$ It is conventional to set $p+q=2 n$.

3 The Atiyah-Ward space-time of signature $(+,+,-,-)$ is also possible [12].
} 
The $C^{\alpha \beta} \neq 0$ explicitly break the four-dimensional Euclidean invariance. The NAC nature of $\theta$ 's can be fully taken into account by using the (associative, but non-commutatvive) Moyal-Weyl-type (star) product of superfields,

$$
f(\theta) * g(\theta)=f(\theta) \exp \left(-\frac{C^{\alpha \beta}}{2} \frac{\overleftarrow{\partial}}{\partial \theta^{\alpha}} \frac{\partial}{\partial \theta^{\beta}}\right) g(\theta)
$$

which respects the $\mathrm{N}=1$ superspace chirality. ${ }^{4}$ The star product (1.31) is polynomial in the deformation parameter,

$$
f(\theta) * g(\theta)=f g+(-1)^{\operatorname{deg} f} \frac{C^{\alpha \beta}}{2} \frac{\partial f}{\partial \theta^{\alpha}} \frac{\partial g}{\partial \theta^{\beta}}-\operatorname{det} C \frac{\partial^{2} f}{\partial \theta^{2}} \frac{\partial^{2} g}{\partial \theta^{2}},
$$

where we have used the identity

$$
\operatorname{det} C=\frac{1}{2} \varepsilon_{\alpha \gamma} \varepsilon_{\beta \delta} C^{\alpha \beta} C^{\gamma \delta},
$$

and the notation

$$
\frac{\partial^{2}}{\partial \theta^{2}}=\frac{1}{4} \varepsilon^{\alpha \beta} \frac{\partial}{\partial \theta^{\alpha}} \frac{\partial}{\partial \theta^{\beta}}
$$

We also use the following book-keeping notation for 2-component spinors:

$$
\theta \chi=\theta^{\alpha} \chi_{\alpha}, \quad \bar{\theta} \bar{\chi}=\bar{\theta}_{\dot{\alpha}} \bar{\chi}^{\dot{\alpha}}, \quad \theta^{2}=\theta^{\alpha} \theta_{\alpha}, \quad \bar{\theta}^{2}=\bar{\theta}_{\dot{\alpha}} \bar{\theta}^{\dot{\alpha}} .
$$

The spinorial indices are raised and lowered by the use of two-dimensional Levi-Civita symbols. Grassmann integration amounts to Grassmann differentiation. The anti-chiral covariant derivative in the chiral superspace basis is $\bar{D}_{\dot{\alpha}}=-\partial / \partial \bar{\theta}^{\dot{\alpha}}$. The field component expansion of a chiral superfield $\Phi$ reads

$$
\Phi(y, \theta)=\phi(y)+\sqrt{2} \theta \chi(y)+\theta^{2} M(y) .
$$

An anti-chiral superfield $\bar{\Phi}$ in the chiral basis is given by

$$
\begin{aligned}
\bar{\Phi}\left(y^{\mu}-2 i \theta \sigma^{\mu} \bar{\theta}, \bar{\theta}\right)= & \bar{\phi}(y)+\sqrt{2} \bar{\theta} \bar{\chi}(y)+\bar{\theta}^{2} \bar{M}(y) \\
& +\sqrt{2} \theta\left(i \sigma^{\mu} \partial_{\mu} \bar{\chi}(y) \bar{\theta}^{2}-i \sqrt{2} \sigma^{\mu} \bar{\theta} \partial_{\mu} \bar{\phi}(y)\right)+\theta^{2} \bar{\theta}^{2} \triangle \bar{\phi}(y)
\end{aligned}
$$

where $\triangle=\partial_{\mu} \partial_{\mu}$. The bars over fields serve to distinguish between the 'left' and 'right' components that are truly independent in Euclidean space.

The non-anticommutativity $C_{\alpha \beta} \neq 0$ also explicitly breaks half of the original $\mathrm{N}=1$ supersymmetry [13]. Only the chiral subalgebra generated by the chiral supercharges (in the chiral basis) $Q_{\alpha}=\partial / \partial \theta^{\alpha}$ is preserved, with $\left\{Q_{\alpha}, Q_{\beta}\right\}_{*}=0$, thus defining what is now called $\mathrm{N}=1 / 2$ supersymmetry. The

${ }^{4}$ We use the left derivatives as a default, the right ones are explicitly indicated. 
use of the NAC-deformed superspace allows one to keep $\mathrm{N}=1 / 2$ supersymmetry manifest. The $\mathrm{N}=1 / 2$ supersymmetry transformation laws of the chiral and anti-chiral superfield components in eqs. (1.36) and (1.37) are as follows:

$$
\delta \phi=\sqrt{2} \varepsilon^{\alpha} \chi_{\alpha}, \quad \delta \chi_{\alpha}=\sqrt{2} \varepsilon_{\alpha} M, \quad \delta M=0,
$$

and

$$
\delta \bar{\phi}=0, \quad \delta \bar{\chi}^{\dot{\alpha}}=-i \sqrt{2}\left(\tilde{\sigma}_{\mu}\right)^{\dot{\alpha} \beta} \varepsilon_{\beta} \partial_{\mu} \bar{\phi}, \quad \delta \bar{M}=-i \sqrt{2} \partial_{\mu} \bar{\chi}_{\dot{\alpha}}\left(\tilde{\sigma}_{\mu}\right)^{\dot{\alpha} \beta} \varepsilon_{\beta},
$$

respectively, where we have introduced the $\mathrm{N}=1 / 2$ supersymmetry (chiral) parameter $\varepsilon^{\alpha}$.

To the end of this section, we are going to demonstrate that, in the case of the supersymmetric NLSM, its NAC superworldvolume gives rise to the induced smearing or fuzzyness in the NLSM target space $[14,15]$. Here we follow ref. [15] where the most general four-dimensinal supersymmetric NLSM with an arbitrary scalar potential in the NAC superspace was considered (without any gauge fields), with the action

$$
S[\Phi, \bar{\Phi}]=\int d^{4} y\left[\int d^{2} \theta d^{2} \bar{\theta} K\left(\Phi^{i}, \bar{\Phi}^{\bar{j}}\right)+\int d^{2} \theta W\left(\Phi^{i}\right)+\int d^{2} \bar{\theta} \bar{W}\left(\bar{\Phi}^{\bar{j}}\right)\right] .
$$

This action is completely specified by the Kähler superpotential $K(\Phi, \bar{\Phi})$, the scalar superpotential $W(\Phi)$, and the anti-chiral superpotential $\bar{W}(\bar{\Phi})$, in terms of some number $n$ of chiral and anti-chiral superfields, $i, \bar{j}=1,2, \ldots, n$. In Euclidean superspace the functions $W(\Phi)$ and $\bar{W}(\bar{\Phi})$ are independent upon each other. The NAC-deromed action is formally obtained by replacing all superfield profucts in eq. (1.40) by their star products (1.31).

The NAC-deformed extension of eq. (1.40) in four dimensions after a 'Seiberg-Witten map' (i.e. after an explicit computation of all star products) was found in a closed form (i.e. in terms of finite functions) in refs. [15, 16, 17]. Our four-dimensional results are in agreement with the results of ref. [14] in the case of the NAC-deformed $\mathrm{N}=2$ supersymmetric two-dimensionl NLSM, after dimensional reduction to two dimensions.

We use the following notation valid for any function $F(\phi, \bar{\phi})$ :

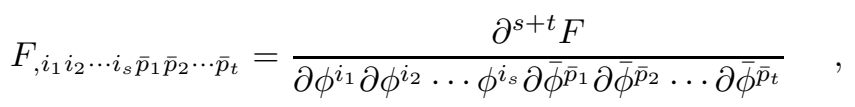

and the Grassmann integral normalisation $\int d^{2} \theta \theta^{2}=1$. The actual deformation parameter, in the case of the NAC-deformed field theory (1.40), appears to be

$$
c=\sqrt{-\operatorname{det} C},
$$

where we have used the definition [13]

$$
\operatorname{det} C=\frac{1}{2} \varepsilon_{\alpha \gamma} \varepsilon_{\beta \delta} C^{\alpha \beta} C^{\gamma \delta} .
$$


As a result, unlike the case of the NAC-deformed supersymmetric gauge theories [13], the NAC-deformation of the NLSM field theory (1.40) appears to be invariant under Euclidean translations and rotations.

A simple non-perturbative formula, describing an arbitrary NAC-deformed scalar superpotential $V$ depending upon a single chiral superfield $\Phi$, was found in ref. [16],

$$
\begin{aligned}
\int d^{2} \theta V_{*}(\Phi)= & \frac{1}{2 c}[V(\phi+c M)-V(\phi-c M)] \\
& -\frac{\chi^{2}}{4 c M}\left[V_{, \phi}(\phi+c M)-V_{, \phi}(\phi-c M)\right] .
\end{aligned}
$$

The NAC-deformation in the single superfield case thus gives rise to the split of the scalar potential, which is controlled by the auxiliary field $M$. When using an elementary identity

$$
f(x+a)-f(x-a)=a \frac{\partial}{\partial x} \int_{-1}^{+1} d \xi f(x+\xi a),
$$

valid for any function $f$, we can rewrite eq. (1.44) to the equivalent form [14]

$$
\int d^{2} \theta V_{*}(\Phi)=\frac{1}{2} M \frac{\partial}{\partial \phi} \int_{-1}^{+1} d \xi V(\phi+\xi c M)-\frac{1}{4} \chi^{2} \frac{\partial^{2}}{\partial \phi^{2}} \int_{-1}^{+1} d \xi V(\phi+\xi c M) .
$$

Similarly, in the case of several chiral superfields, one finds [14]

$$
\int d^{2} \theta V_{*}\left(\Phi^{I}\right)=\frac{1}{2} M^{I} \frac{\partial}{\partial \phi^{I}} \widetilde{V}(\phi, M)-\frac{1}{4}\left(\chi^{I} \chi^{J}\right) \frac{\partial^{2}}{\partial \phi^{I} \partial \phi^{J}} \widetilde{V}(\phi, M)
$$

in terms of the auxiliary pre-potential

$$
\widetilde{V}(\phi, M)=\int_{-1}^{+1} d \xi V\left(\phi^{I}+\xi c M^{I}\right) .
$$

Hence the NAC-deformation of a generic scalar superpotential $V$ results in its smearing or fuzziness controlled by the auxiliary fields $M^{I}$.

A calculation of the NAC deformed Kähler potential

$$
\int d^{4} y L_{\text {kin. }} \equiv \int d^{4} y \int d^{2} \theta d^{2} \bar{\theta} K\left(\Phi^{i}, \bar{\Phi}^{\bar{j}}\right)_{*}
$$

can be reduced to eqs. (1.44) or (1.47), when using a chiral reduction in superspace, with the following result [17]:

$$
\begin{aligned}
L_{\text {kin. } .=} & \frac{1}{2} M^{i} Y_{, i}+\frac{1}{2} \partial^{\mu} \bar{\phi}^{\bar{p}} \partial_{\mu} \bar{\phi}^{\bar{q}} Z_{, \bar{p} \bar{q}}+\frac{1}{2} \triangle \bar{\phi}^{\bar{p}} Z_{, \bar{p}}-\frac{1}{4}\left(\chi^{i} \chi^{j}\right) Y_{, i j} \\
& -\frac{1}{2} i\left(\chi^{i} \sigma^{\mu} \bar{\chi}^{\bar{p}}\right) \partial_{\mu} \bar{\phi}^{\bar{q}} Z_{, i \bar{p} \bar{q}}-\frac{1}{2} i\left(\chi^{i} \sigma^{\mu} \partial_{\mu} \bar{\chi}^{\bar{p}}\right) Z_{, i \bar{p}},
\end{aligned}
$$


where we have introduced the (component) smeared Kähler pre-potential

$$
Z(\phi, \bar{\phi}, M)=\int_{-1}^{+1} d \xi K^{\xi} \quad \text { with } \quad K^{\xi} \equiv K\left(\phi^{i}+\xi c M^{i}, \bar{\phi}^{\bar{j}}\right)
$$

as well as the extra (auxiliary) pre-potential [14]

$Y(\phi, \bar{\phi}, M, \bar{M})=\bar{M}^{\bar{p}} Z_{, \bar{p}}-\frac{1}{2}\left(\bar{\chi}^{\bar{p}} \bar{\chi}^{\bar{q}}\right) Z_{, \bar{p} \bar{q}}+c \int_{-1}^{+1} d \xi \xi\left[\partial^{\mu} \bar{\phi}^{\bar{p}} \partial_{\mu} \bar{\phi}^{\bar{q}} K_{, \bar{p} \bar{q}}^{\xi}+\triangle \bar{\phi}^{\bar{p}} K_{, \bar{p}}^{\xi}\right]$

It is not difficult to check that eq. (1.50) does reduce to the standard (Kähler) $\mathrm{N}=1$ supersymmetric NLSM (cf. sect. 4) in the limit $c \rightarrow 0$. Also, in the case of a free (bilinear) Kähler potential $K=\delta_{i \bar{j}} \Phi^{i} \bar{\Phi}^{\bar{j}}$, there is no deformation at all.

The NAC-deformed scalar superpotentials $W(\Phi)_{*}$ and $\bar{W}(\bar{\Phi})_{*}$ imply, via eqs. (1.47) and (1.48), that the following component terms are to be added to eq. (1.50):

$$
L_{\text {pot. }}=\frac{1}{2} M^{i} \widetilde{W}_{, i}-\frac{1}{4}\left(\chi^{i} \chi^{j}\right) \widetilde{W}_{, i j}+\bar{M}^{\bar{p}} \bar{W}_{, \bar{p}}-\frac{1}{2}\left(\bar{\chi}^{\bar{p}} \bar{\chi}^{\bar{q}}\right) \bar{W}_{, \bar{p} \bar{q}},
$$

where we have introduced the smeared scalar pre-potential [14]

$$
\widetilde{W}(\phi, M)=\int_{-1}^{+1} d \xi W\left(\phi^{i}+\xi c M^{i}\right) .
$$

The anti-chiral superpotential terms are inert under the NAC-deformation.

The $\xi$-integrations in the equations above represent the smearing effects. However, the smearing is merely apparent in the case of a single chiral superfield, which gives rise to the splitting (1.44) only. This can also be directly demonstrated from eq. (1.50) when using the identity (1.45) together with the related identity [17]

$$
f(x+a)+f(x-a)=\int_{-1}^{+1} d \xi f(x+\xi a)+a \frac{\partial}{\partial x} \int_{-1}^{+1} d \xi \xi f(x+\xi a) .
$$

The single superfield case thus appears to be special, so that a sum of eq. (1.50) and (1.53) can be rewritten to the bosonic contribution [17]

$$
\begin{aligned}
L_{\mathrm{bos.}}= & +\frac{1}{2} \partial^{\mu} \bar{\phi} \partial_{\mu} \bar{\phi}\left[K_{, \bar{\phi} \bar{\phi}}(\phi+c M, \bar{\phi})+K_{, \bar{\phi} \bar{\phi}}(\phi-c M, \bar{\phi})\right] \\
& +\frac{1}{2} \triangle \bar{\phi}\left[K_{, \bar{\phi}}(\phi+c M, \bar{\phi})+K_{, \bar{\phi}}(\phi-c M, \bar{\phi})\right] \\
& +\frac{\bar{M}}{2 c}\left[K_{, \bar{\phi}}(\phi+c M, \bar{\phi})-K_{, \bar{\phi}}(\phi-c M, \bar{\phi})\right] \\
& +\frac{1}{2 c}[W(\phi+c M)-W(\phi-c M)]+\bar{M} \frac{\partial \bar{W}}{\partial \bar{\phi}}
\end{aligned}
$$


supplemented by the following fermionic terms [17]:

$$
\begin{aligned}
L_{\mathrm{ferm} .}= & -\frac{1}{4 c} \bar{\chi}^{2}\left[K_{, \bar{\phi} \bar{\phi}}(\phi+c M, \bar{\phi})-K_{, \bar{\phi} \bar{\phi}}(\phi-c M, \bar{\phi})\right] \\
& -\frac{i}{2 c M}\left(\chi \sigma^{\mu} \bar{\chi}\right) \partial_{\mu} \bar{\phi}\left[K_{, \bar{\phi} \bar{\phi}}(\phi+c M, \bar{\phi})-K_{, \bar{\phi} \bar{\phi}}(\phi-c M, \bar{\phi})\right] \\
& -\frac{i}{2 c M}\left(\chi \sigma^{\mu} \partial_{\mu} \bar{\chi}\right)\left[K_{, \bar{\phi}}(\phi+c M, \bar{\phi})-K_{, \bar{\phi}}(\phi-c M, \bar{\phi})\right] \\
& -\frac{\bar{M}}{4 c M} \chi^{2}\left[K_{, \phi \bar{\phi}}(\phi+c M, \bar{\phi})-K_{, \phi \bar{\phi}}(\phi-c M, \bar{\phi})\right] \\
& -\frac{1}{4 M} \chi^{2} \partial^{\mu} \bar{\phi} \partial_{\mu} \bar{\phi}\left[K_{, \phi \bar{\phi} \bar{\phi}}(\phi+c M, \bar{\phi})+K_{, \phi \bar{\phi} \bar{\phi}}(\phi-c M, \bar{\phi})\right] \\
& +\frac{1}{4 c M^{2}} \chi^{2} \partial^{\mu} \bar{\phi} \partial_{\mu} \bar{\phi}\left[K_{, \bar{\phi} \bar{\phi}}(\phi+c M, \bar{\phi})-K_{, \bar{\phi} \bar{\phi}}(\phi-c M, \bar{\phi})\right] \\
& -\frac{1}{4 M} \chi^{2} \triangle \bar{\phi}\left[K_{, \phi \bar{\phi}}(\phi+c M, \bar{\phi})+K_{, \phi \bar{\phi}}(\phi-c M, \bar{\phi})\right] \\
& +\frac{1}{4 c M^{2}} \chi^{2} \triangle \bar{\phi}\left[K_{, \bar{\phi}}(\phi+c M, \bar{\phi})-K_{, \bar{\phi}}(\phi-c M, \bar{\phi})\right] \\
& +\frac{1}{8 c M} \chi^{2} \bar{\chi}^{2}\left[K_{, \phi \bar{\phi} \bar{\phi}}(\phi+c M, \bar{\phi})-K_{, \phi \bar{\phi} \bar{\phi}}(\phi-c M, \bar{\phi})\right] \\
& -\frac{1}{4 c M} \chi^{2}\left[W_{, \phi}(\phi+c M)-W_{, \phi}(\phi-c M)\right]-\frac{1}{2} \bar{\chi}^{2} \bar{W}_{, \bar{\phi} \bar{\phi}} .
\end{aligned}
$$

The anti-chiral auxiliary fields $\bar{M}^{\bar{p}}$ enter the action (1.50) linearly (as Lagrange multipliers), while their algebraic equations of motion,

$$
\frac{1}{2} M^{i} Z_{, i \bar{p}}-\frac{1}{4}\left(\chi^{i} \chi^{j}\right) Z_{, i j \bar{p}}+\bar{W}_{, \bar{p}}=0,
$$

are the non-linear set of equations on the auxiliary fields $M^{i}=M^{i}(\phi, \bar{\phi}) .{ }^{5}$ As a result, the bosonic scalar potential in components is given by [17]

$$
V_{\text {scalar }}(\phi, \bar{\phi})=\left.\frac{1}{2} M^{i} \widetilde{W}_{, i}\right|_{M=M(\phi, \bar{\phi})} .
$$

Some comments are in order.

The NAC-deformation just described is only possible in Euclidean superspace where the chiral and anti-chiral spinors are truly independent.

The NAC-deformed NLSM is completely specified by a Kähler function $K(\Phi, \bar{\Phi})$, a chiral function $W(\Phi)$, an anti-chiral function $\bar{W}(\bar{\Phi})$ and a constant deformation parameter $c$. As a matter of fact, we didn't really use the constancy of $c$, so our results are valid even for any coordinate-dependent NAC deformation with $c(y)$.

\footnotetext{
${ }^{5}$ Equation (1.58) is not a linear system because the function $Z$ is $M$-dependent.
} 
Solving for the auxiliary fields in eq. (1.50) represents not only a technical but also a conceptual problem because of the smearing effects described by the $\xi$-integrations. To bring the kinetic terms in eqs. (1.50) or (1.56) to the standard NLSM form (i.e. without the second order derivatives), one has to integrate by parts that leads to the appearance of the derivatives of the auxiliary fields. This implies that one has to solve eq. (1.58) before integration by parts. Let $M^{i}=M^{i}(\phi, \bar{\phi})$ be a solution to eq. (1.58), and let's ignore fermions for simplicity $\left(\chi_{\alpha}^{i}=\bar{\chi}_{\dot{\alpha}}^{\bar{p}}=0\right)$. Substituting the auxiliary field solution back to the Lagrangian (1.50) and integrating by parts yield

$$
\begin{aligned}
L_{\text {kin. }}(\phi, \bar{\phi})= & -\frac{1}{2}\left(\partial_{\mu} \bar{\phi}^{\bar{p}} \partial_{\mu} \phi^{q}\right) \int_{-1}^{+1} d \xi\left[K_{, \bar{p} q}^{\xi}+2 c \xi M_{, q}^{i} K_{, \bar{p} i}^{\xi}+c \xi M^{i} K_{, \bar{p} i q}^{\xi}\right. \\
& \left.+c^{2} \xi^{2} M^{i} K_{, \bar{p} i j}^{\xi} M_{, q}^{j}\right] \\
& -\frac{1}{2}\left(\partial_{\mu} \bar{\phi}^{\bar{p}} \partial_{\mu} \bar{\phi}^{\bar{q}}\right) \int_{-1}^{+1} d \xi\left[2 c \xi K_{, \bar{p} i}^{\xi} M_{, \bar{q}}^{i}+c^{2} \xi^{2} M^{i} K_{, \bar{p} i j}^{\xi} M_{, \bar{q}}^{j}\right] .
\end{aligned}
$$

It is now apparent that the NAC-deformation does not preserve the original Kähler geometry of eq. (1.40), though the absence of $\left(\partial_{\mu} \phi\right)^{2}$ terms and the particular structure of various contributions to eq. (1.60) are quite remarkable. The action (1.60) takes the form of a generic NLSM, being merely dependent upon mixed derivatives of the Kähler function, so that the original Kähler gauge invariance of eq. (1.40),

$$
K(\phi, \bar{\phi}) \rightarrow K(\phi, \bar{\phi})+f(\phi)+\bar{f}(\bar{\phi})
$$

with arbitrary gauge functions $f(\phi)$ and $\bar{f}(\bar{\phi})$ is preserved. See ref. [16] for more discussion about elimination of the auxiliary fields.

As a result, the NAC deformation of the NLSM (1.40) amounts to the non-Kählerian and non-Hermitian deformation of the original Kählerian and Hermitian structures, which is controlled by the auxiliary field solution to eq. (1.58). In the case of a single chiral superfield, the deformed NLSM metric can be read off from the following kinetic terms [16]:

$$
\begin{aligned}
L_{\mathrm{kin} .}(\phi, \bar{\phi})= & -\frac{1}{2}\left(\partial_{\mu} \bar{\phi} \partial_{\mu} \phi\right) \frac{\partial}{\partial \phi} \frac{\partial}{\partial \bar{\phi}}[K(\phi+c M(\phi, \bar{\phi}), \bar{\phi})+K(\phi-c M(\phi, \bar{\phi}), \bar{\phi})] \\
& +\frac{1}{2}\left(\partial_{\mu} \bar{\phi} \partial_{\mu} \phi\right) \frac{\partial}{\partial \phi}\left[c K_{, \phi}(\phi+c M, \bar{\phi})-c K_{, \phi}(\phi-c M, \bar{\phi})\right] \frac{\partial M(\phi, \bar{\phi})}{\partial \bar{\phi}} \\
& -\frac{1}{2}\left(\partial_{\mu} \bar{\phi} \partial_{\mu} \bar{\phi}\right)\left[c K_{, \phi \bar{\phi}}(\phi+c M(\phi, \bar{\phi}), \bar{\phi})-c K_{, \phi \bar{\phi}}(\phi-c M(\phi, \bar{\phi}), \bar{\phi})\right] \\
& \times \frac{\partial M(\phi, \bar{\phi})}{\partial \bar{\phi}} .
\end{aligned}
$$


In the case of several superfields, the deformed NLSM can be read off from eq. (1.60), when assuming all the $\xi$-integrations to be performed with the auxiliary fields considered as spectators.

We thus find a new (NAC) mechanism of deformation of complex geometry in the supersymmetric NLSM target space, by using a non-vanishing anti-holomorphic scalar potential $\bar{W}(\bar{\Phi})$, because elimination of the auxiliary fields $M$ and $\bar{M}$ via their algebraic equations of motion in the NAC deformed NLSM results in the deformed bosonic Kähler potential depending upon $C$ and $\bar{W}^{\prime}(\bar{\Phi})$. This feature is specific to the NAC deformation, because a scalar potential does not affect a Kähler potential in the usual (undeformed) NLSM.

\subsection{Example: NAC-deformed $C P(1)$ model}

Let's consider the simplest non-trivial example provided by a four-dimensional supersymmetric $C P(1)$ NLSM with the (undeformed) Kähler, Hermitian and symmetric target space characterized by the Kähler potential

$$
K(\phi, \bar{\phi})=\alpha \ln \left(1+\kappa^{-2} \phi \bar{\phi}\right),
$$

where two dimensional constants, $\alpha$ and $\kappa$, have been introduced, and with an arbitrary anti-holomorphic scalar superpotential $\bar{W}(\bar{\Phi})$. An explicit solution to the auxiliary field equation (1.58) in this case reads [16]

$$
M=\frac{\alpha-\sqrt{\alpha^{2}+\left(2 c \bar{\phi}\left(1+\kappa^{-2} \phi \bar{\phi}\right) \bar{W}_{, \bar{\phi}}\right)^{2}}}{2 c^{2} \kappa^{-2} \bar{\phi}^{2} \bar{W}_{, \bar{\phi}}},
$$

where we have used the notation $\bar{W}_{, \bar{\phi}}=\partial \bar{W} / \partial \bar{\phi}$. A straightforward calculation yields the following deformed NLSM kinetic terms [16]:

$$
L_{\text {kin. }}=-g_{\phi \phi} \partial_{\mu} \phi \partial_{\mu} \phi-2 g_{\phi \bar{\phi}} \partial_{\mu} \phi \partial_{\mu} \bar{\phi}-g_{\bar{\phi} \bar{\phi}} \partial_{\mu} \bar{\phi} \partial_{\mu} \bar{\phi}
$$

where

$$
\begin{aligned}
g_{\phi \bar{\phi}}= & \frac{-\alpha \kappa^{-2} c^{2} \bar{\phi}^{2}\left(\bar{W}_{, \bar{\phi}}\right)^{2}}{\left(-\alpha+\sqrt{\alpha^{2}+\left(2 c \bar{\phi}\left(1+\kappa^{-2} \phi \bar{\phi}\right) \bar{W}_{, \bar{\phi}}\right)^{2}}\right) \sqrt{\alpha^{2}+\left(2 c \bar{\phi}\left(1+\kappa^{-2} \phi \bar{\phi}\right) \bar{W}_{, \bar{\phi}}\right)^{2}}}, \\
g_{\phi \phi}= & 0, \\
g_{\bar{\phi} \bar{\phi}}= & \frac{-2 \alpha^{-1} c^{2}\left(1+\kappa^{-2} \phi \bar{\phi}\right) \bar{W}_{, \bar{\phi}}}{\left(\alpha-\sqrt{\alpha^{2}+\left(2 c \bar{\phi}\left(1+\kappa^{-2} \phi \bar{\phi}\right) \bar{W}_{, \bar{\phi}}\right)^{2}}\right) \sqrt{\alpha^{2}+\left(2 c \bar{\phi}\left(1+\kappa^{-2} \phi \bar{\phi}\right) \bar{W}_{, \bar{\phi}}\right)^{2}}} \times \\
& \times\left[4 c^{2} \bar{\phi}^{2}\left(\bar{W}_{, \bar{\phi}}\right)^{3}\left(1+\kappa^{-2} \phi \bar{\phi}\right)\right. \\
& \left.+\alpha\left(\alpha-\sqrt{\alpha^{2}+\left(2 c \bar{\phi}\left(1+\kappa^{-2} \phi \bar{\phi}\right) \bar{W}_{, \bar{\phi}}\right)^{2}}\right)\left(2 \bar{W}_{, \bar{\phi}}+\bar{\phi} \bar{W}_{, \bar{\phi} \bar{\phi}}\right)\right] .
\end{aligned}
$$


It is worth noticing that $\operatorname{det} g=-\left(g_{\phi \bar{\phi}}\right)^{2}$. The most apparent feature $g_{\phi \phi}=0$ is also valid in the case of a generic NAC-deformed NLSM (in the given parametrization).

\subsection{Conclusion}

Our approach to the NAC-deformed NLSM is very general. The NAC deformation (i.e. smearing or fuzziness) of the NLSM Kähler potential is controlled by the auxiliary fields $M^{i}$ entering the deformed Kähler potential in the highly non-linear way. Both locality and Euclidean invariance are preserved, while no higher derivatives appear in the deformed NLSM action.

One should distinguish between the NAC-deformation and $\mathrm{N}=1 / 2$ supersymmetry. Though the NAC-deformation we considered is $\mathrm{N}=1 / 2$ supersymmetric, the former is stronger than the latter. When requiring merely $\mathrm{N}=1 / 2$ supersymmetry of a four-dimensional NLSM, it would give rise to much weaker restrictions on the NLSM target space.

It is still the open question how to describe the NAC deformation of the NLSM metric in purely geometrical terms.

This investigation was supported in part by the Japanese Society for Promotion of Science (JSPS). I am grateful to K. Ito, O. Lechtenfeld, P. Bowknegt and S. Watamura for useful discussions during the workshop.

\section{References}

1. Ketov S.V.: Quantum Non-Linear Sigma Models. Springer-Verlag, Berlin Heidelberg New-York (2000)

2. Spindel, P., Sevrin, A., Troost, W., van Proeyen, A.: Extended Supersymmetric Sigma Models on Group Manifolds. Nucl. Phys. B308, 662-698 (1988), and B311, 465-492 (1988)

3. Sevrin, A., Troost J.: Off-shell Formulation of $\mathrm{N}=2$ Non-linear Sigma-models. Nucl. Phys. B492, 623-646 (1997)

4. Huybrechts D.: Complex Geometry. Springer-Verlag, Berlin Heidelberg NewYork (2004)

5. Gates, S.J. Jr., Hull, C., Roček, M.: Twisted Multiplets and New Supersymmetric Non-linear Sigma Models. Nucl. Phys. B248, 157-186 (1984)

6. Buscher, T., Lindström, U., Roček, M.: New Supersymmetric Sigma-models with Wess-Zumino Terms. Phys. Lett. B202, 94-98 (1988)

7. Gates, S.J. Jr., Ketov, S.V.: No N=4 Strings on Wolf Spaces. Phys. Rev. D52, 2278-2293 (1995)

8. Galperin, A., Ivanov, E., Ogievetsky, V., Sokatchev, E.: Harmonic Superspace. Cambridge University Press (2001)

9. Brink, L., Schwarz J.: Quantum Superspace. Phys.Lett. B100, 310-312 (1981)

10. Klemm, D., Penati, S., Tamassia, L.: Non(anti)commutative Superspace. Classical and Quantum Grav. 20, 2905-2916 (2003) 
11. Ooguri, H., Vafa C.: Gravity induced C Deformation. Adv. Theor. Math. Phys. 7, 405-417 (2004)

12. Gates, S.J. Jr., Ketov, S.V., Nishino, H.: Self-dual Supersymmetry and Supergravity in Atiyah-Ward Space-time. Nucl. Phys. B716, 149-210 (1993)

13. Seiberg, N.: Noncommutative Superspace, $N=1 / 2$ Supersymmetry and String Theory. JHEP 0306, 010 (2003)

14. Alvarez-Gaume, L., Vazquez-Mozo, M.: On Non-anti-commutative N=2 Sigmamodels in Two Dimensions. JHEP 0504, 007 (2005)

15. Hatanaka, T., Ketov, S.V., Kobayashi, Y., Sasaki, S.: N=1/2 Supersymmetric Four-dimensional Non-linear $\sigma$-models from Non-anti-commutative Superspace. Nucl. Phys. B726, 481-493 (2005)

16. Hatanaka, T., Ketov, S.V., Kobayashi, Y., Sasaki, S.: Non-anti-commutative Deformation of Effective Potentials in Supersymmetric Gauge Theories. Nucl. Phys. B716, 88-104 (2005)

17. Hatanaka, T., Ketov, S.V., Sasaki, S.: Summing up Non-anti-commutative Kähler Potential. Phys. Lett. B619, 352-358 (2005). 